doi $10.34074 /$ scop. 3005007

\title{
COLLABORATION BETWEEN CLINICAL PRACTICE PROVIDERS AND A SCHOOL OF NURSING: NOW IS GOOD
}

\author{
Ruth Crawford, Lyn Wardlaw, Nicola Hughes and Helen Lelean
}

\section{INTRODUCTION}

Nursing education prepares undergraduate students for a nursing career and clinical practice providers provide the career opportunities. That much is true, but as we emerge from the COVID-19 pandemic, it has become clear that a theory-practice gap still exists. In this opinion piece, we provide a success story which demonstrates how a collaboration between nursing education and practice is reducing the theory-practice gap.

In the past, nurses were educated in hospitals, through an apprenticeship system which met the needs of the hospital and health providers, but frequently put patient needs before student learning. That all changed in Aotearoa/New Zealand in the 1970s and 1980s following a report (Carpenter, 197I) which sharply criticised this model and recommended moving the education of registered nurses away from health providers and into tertiary institutions. As a consequence of this move, the health care system became distanced from involvement in the development of nursing curricula. This was problematic when schools of nursing were preparing students for a career in that health system. When health care providers are removed from the day-to-day organisation of nursing education and nursing education providers move away from patient management, there becomes a divide, leading to nursing staff to sometimes being unwelcoming to students and graduating students struggling to "hit the ground running'.

In Taranaki, New Zealand, we are working hard to change that model and to improve communication between nursing education and nursing practice provision. A new Director of Nursing at the Taranaki District Health Board (TDHB) and a new Director of the School at the local nursing education provider, the Western Institute of Technology (WITT), have combined forces. They are collaborating to develop WITT nursing graduates who are socialised into nursing practice and supported in their education as they begin their nursing career. Many of those nursing graduates are the future employees of TDHB so it is in everyone's interest that education and health provision work together.

There have been a number of initiatives over the past year to increase collaboration. In mid-2019, a Joint Clinical Monitoring Committee (JCMC) was established to increase communication and collaboration, meeting twomonthly to discuss curriculum, student and career planning issues that arise. TDHB appointed a Nurse Educator who focuses on undergraduate nursing education and Nurse Entry to Practice (NEtP) graduates, and WITT appointed a Lead, Nursing and Mental Health, to coordinate and liaise with TDHB to ensure that the curriculum is responsive to practice. In October 2019, WITT adopted a new undergraduate nursing curriculum, developed by the Ara Institute of Technology. With the support of Ara and TDHB, the curriculum was implemented to commence in 2020 for Year One and Two nursing students. 
In late 2019, the Chief Executive of WITT and the Chief Executive of TDHB developed a Memorandum of Understanding $(\mathrm{MoU})$, agreeing to establish an enduring partnership between the TDHB and WITT, and allowing for the exchange of information, experience and expertise. WITT are committed to producing the best nurses for Taranaki and beyond, and TDHB want to give those nurses the best start, providing the region with an even more capable health care workforce. The purpose of the MoU is to establish mutual trust, conventions and tacit understanding; the basis of a strategic cooperative partnership. From the $\mathrm{MoU}$ and collaboration between Directors and lead staff, in mid-2020 WITT and TDHB are establishing Dedicated Education Units (DEUs), clinical areas where student learning is at the forefront, and clinical staff have opportunity to be engaged and active in student learning.

Collaboration cannot be taken for granted; like any relationship it has to be consciously worked on and nurtured. For this article, the two Directors (Lyn Wardlaw (LW), Director of Nursing, TDHB, and Ruth Crawford (RC), Director, School of Nursing, Health and Wellness, WITT) and the two Leads in nursing and education (Nicola Hughes (NH), Nurse Educator, TDHB, and Helen Lelean ( $\mathrm{HL})$, Lead, Nursing and Mental Health, WITT) independently responded to specific questions about collaboration between education and practice. Responses to the questions are identified from each respondent, to enable the reader to gain an understanding of collaboration from the perspective of each respondent.

\section{What do you understand by the term 'collaboration' in relation to nursing education?}

LW (practice): If nurses are to be as effective as possible in helping to provide high-quality patient care, they'll need to be better prepared as care becomes more complex and shifts to the home and community. We also need more nurses with advanced degrees to provide primary care and to teach the next generation of nurses. A bachelor's degree in nursing best prepares nurses to take on increasingly complex roles.

Studies have demonstrated how effective coordination and communication among health professionals can enhance the quality and safety of patient care. Health professionals working collaboratively as integrated teams draw on individual and collective skills and experience across disciplines.

RC (education): Nursing education has to be a team effort; it takes a community to grow a nurse so collaboration between education and practice is vital to ensuring our graduates are prepared for the world of nursing practice. As educators, we need to ensure that nursing education is relevant, evidence-based and future focused. Collaboration not only with health providers, but with regulators (Nursing Council of New Zealand) and other education providers in New Zealand and around the world, ensure we are educating nurses for the practice of nursing, wherever they may choose to go.

$\mathrm{NH}$ (practice): Collaboration is about working together to achieve a common goal. It requires open communication, trust and appreciation for diversity of ideas. In regards to nursing education our main goal is to ensure nurses work together with students to provide them with learning opportunities and support them to grow and develop their practice and assist in linking theory to practice in a clinical setting.

HL (education): Working together with stakeholders to provide the best possible clinical experience for nursing students. The ultimate aim is to collaborate effectively with members of the healthcare team for all clinical areas e.g. community and hospital.

\section{What is the primary role of nursing practice providers (such as TDHB) in undergraduate nursing education?}

LW: Learning skills to effectively work in teams so they can provide collaborative, safe, high-quality, accessible patient-centred care. 
RC: Nursing education must be grounded in nursing practice, so practice has a pivotal role in providing students the opportunity to practise the theory and competencies they have learnt in the classroom and simulation. Nursing practice providers need to give students learning opportunities so they can safely and securely practise their learning in a supportive, nurturing environment. The nursing profession must take control of the education of future nurses, thus ensuring that practice changes are embedded into curriculum.

$\mathrm{NH}$ : Our primary role from TDHB is to support and facilitate learning opportunities for the nursing students in a variety of clinical settings. They are our future nurses and we want to grow and foster them to ensure they are as workforce ready as possible when they are qualified. We maintain open communication channels with WITT to ensure we are providing consistent messages to all staff and students.

HL: To provide the best possible clinical experience for undergraduate nursing students. This should include preceptorship and a supportive learning environment which will help to prepare nursing students for the role of Registered Nurse (RN).

\section{What is the primary role of nursing education providers (such as WITT) in undergraduate nursing education?}

LW: To ensure that all nursing students are work ready to begin their nursing careers, that being said, to ensure the nurse knows when to escalate; knows their own abilities, has insight and is able to critically think within their scope as new learners.

RC: Education providers have a responsibility to ensure the education they are providing is relevant, evidencebased, and will prepare students for nursing into the future. Undergraduate education is just the beginning for nurses; we need to ensure they become lifelong learners, constantly adapting and changing to the needs of health care systems and populations. Education is preparing students to practice inter-professionally with a diverse population.

$\mathrm{NH}$ : To support students with their academic learning and substantially strengthen and embed evidence-based practice learning and research. WITT also work together with TDHB staff to provide appropriate learning environments for students.

HL: To provide the most up-to-date evidenced-based nursing knowledge and support them throughout the nursing programme to achieve their potential of becoming a Registered Nurse. This will also include providing pastoral and academic support.

\section{What changes have occurred between WITT and TDHB which have enabled/facilitated collaboration in undergraduate nursing education?}

LW: Better communication, team work and a central desired outcome to have work-ready nurses as new practitioners.

RC: The biggest change is the development of a trusting relationship between education and practice. Education trusts that the DHB will provide a quality learning experience and that student's learning needs will be considered when they are on placement and the DHB trusts that WITT will provide students who are well prepared for clinical practice, eager to learn from RNs and other inter-professional staff, and willing to take feedback on their performance.

$\mathrm{NH}$ : There have been newly appointed people into key positions that influence the working relationships between WITT and TDHB. New ideas and perspectives have been introduced and there is a real sense of working together to address any concerns with the common goal of supporting our students and nursing staff. 
HL: A change of Director of Nursing at both TDHB and WITT, as well as the CEOs working together to sign a Memorandum of Understanding, has improved the relationship and collaboration between both areas. A DEU model is planned for next semester which has also enabled more facilitation between WITT and TDHB.

\section{What hinders collaboration between nursing education providers (WITT) and nursing practice providers (TDHB)?}

LW: Poor communication and differing visions.

RC: Poor communication and misunderstandings could hinder collaboration; it is vital that we continue to discuss practice and education concerns, that we share ideas, listen and support each other, and constantly check we have a common vision and purpose.

$\mathrm{NH}$ : I wouldn't say hinders but what may cause confusion initially is not knowing the expectations/roles they have of each other. There is also an element of resistance at times from the ward staff not being fully aware of what a student can and cannot do. This has a flow-on effect for the students who can feel like a burden.

HL: The change of curriculum has the potential to cause issues, for example Preceptor requirements have changed and staff (RNs) are not totally familiar with the requirements as yet.

\section{What enhances collaboration between nursing education (WITT) and nursing practice (TDHB)?}

LW: Opposite of the above $=$ good communication and the same vision.

RC: What has worked is sharing ideas, discussing issues as they arise, providing informal and formal opportunities to meet. I have valued monthly informal catch ups with LW where we can discuss what is going on and plan the future, as well as the more formal Clinical Monitoring meeting and DEU planning. There is regular communication between $\mathrm{NH}$ and $\mathrm{HL}$ as well, acting on issues of concern, student placement issues, students who need extra support. All these opportunities enhance our collaboration and enables us to work together to a common goal.

$\mathrm{NH}$ : Communication! I cannot emphasise this enough. Without effective communication and sharing of information, there is no collaboration.

HL: Clear communication and regular JCMC/DEU meetings, and also good communication between Directors of Nursing at WITT and TDHB and Lead and Nurse educators. Good communication with the Nursing Lecturers and clinical staff.

\section{How did COVID-19 affect the collaboration between WITT and TDHB in relation to nursing education?}

LW: I believe it enhanced our collaboration for the better.

RC: We had a Clinical Monitoring meeting the day before lockdown and I was impressed at the way TDHB was responding to the situation; calm planning and preparation, ready for whatever came their way. WITT gave all our Personal Protective Equipment to the DHB as at that stage there was uncertainty about their provision. They shared their planning with us which helped us understand the complexity of the situation. Very soon into the lockdown when WITT staff had relocated to their homes, LW contacted me to ask for nursing academics who are able to assist with health provision and we were really pleased to be able to provide support in this way. Pandemic planning has brought us closer together, reminding us that we are all RNs who are ready and able to work together to meet the country's health needs. When we moved to Alert Level 3, the DHB expressed a willingness to place 
students in a variety of clinical environments with a range of support. We were very excited that the DHB would provide this opportunity for students to learn about pandemic health provision.

$\mathrm{NH}$ : It affected the students' placements and made it challenging to accommodate more students in the acute clinical setting due to reduced services/staff. However, both WITT and TDHB worked together to ensure student safety came first; placements were returned to normal when possible and students were provided with education around PPE/COVID-19 and had opportunities to ask any questions to relieve their anxiety about returning to placement.

HL: We were all under pressure but there was clear regular communication throughout COVID-19 and the TDHB were keen and able to still support the students by providing them with placements as soon as they could. Understanding between the two parties was paramount and demonstrated throughout Level 4 down.

\section{What is the future of nursing education collaboration in Taranaki?}

LW: Great nurses for our region.

RC: At WITT, we are very excited about nursing education in Taranaki. We have a positive, constructive relationship with TDHB and other health care providers; we are working with TDHB on establishing Dedicated Education Units together; we are working towards the provision of a health education centre on the hospital site, where nursing students and academics can be based, leading to a closer relationship with health providers. Health providers are sharing with us their feedback of nursing student's preparedness for practice which in turns supports and enhances our curriculum.

$\mathrm{NH}$ : Our exciting new Dedicated Education Unit concept! This will help grow the relationships TDHB have with WITT and provide amazing opportunities for ward staff to be in a position to work with students and foster their learning on a one-to-one basis. The DEU will provide collegial relationships between WITT staff, students and TDHB staff.

HL: Increase in student numbers by improved collaboration with clinical partners for example, external partners, Aged Residential Care, ARC, primary care, mental health. Continued communication with all clinical partners

\section{How can we achieve the future as noted in the question above?}

LW: Same vision, same expectations and working together for the same reason.

RC: By continuing to liaise closely, listening to each other, sharing ideas about the future, hearing the concerns each other has and developing actions which support and develop nursing education in Taranaki.

$\mathrm{NH}$ : By working together to ensure the pilot is successful. Being open minded and considerate to all feedback provided.

HL: Continued good communication and discussions around clinical placements and regular meetings to discuss ways that we can improve the process. 


\section{CONCLUSION}

This paper arose from reflections during the COVID-19 pandemic, specifically on improving the collaborative partnership between nursing education and practice. Collaboration in nursing education provision requires effective communication between health and education providers and a willingness to share ideas. The development of skilled, competent nurses is our collective responsibility; working together we are much more effective than working in silos apart. Inevitably there are always challenges in the partnership as sometimes our needs are different, however we are all committed to working together to overcome these as they arise.

In Taranaki, we are fortunate to have shared aspirations for nursing education and practice; the same expectations and the same vision, working collaboratively to ensure we have excellent nurses to provide nursing care in the region and beyond, into the future. This strategic partnership is enabling practice and education to work together to improve the quality of nursing education and thus, patient outcomes.

Ruth Crawford (100000-000 I-6032-795I) RN, PhD is the Director of the School of Nursing, Health and Wellness at the Western Institute of Technology at Taranaki. Ruth has a background in child health nursing and has been involved in nursing and nursing education for over 30 years.

Lyn Wardlaw RN, RM is the Director of Nursing for Taranaki District Health Board. She previously worked in Australia as a Director of Nursing/Midwifery and Facility Manager for Mareeba District Hospital and also Executive Director of Nursing and Midwifery at Torres \& Cape Hospital Health Service. More recently Lyn was the Operations Manager for Theatres, PACU, Endoscopy, Inventory and CSSD across Waitemata District Health Board.

Nicola Hughes RN is the NETP/NESP Coordinator and Nursing Student Liaison for Taranaki District Health Board. Nicki has a background in medical and surgical nursing throughout a variety of clinical areas in NZ and the UK and has been involved in nursing and nursing education for over 10 years.

Helen Lelean RN, BSc (Hons) Clinical Practice, MEd is the Lead for Nursing \& Mental Health at the Western Institute of Technology at Taranaki. Helen has an extensive clinical background in Medical, Surgical and Intensive Care nursing and has been involved in nursing education both in the UK and NZ for the last 13 years.

Correspondence to: Ruth Crawford, School of Nursing, Health \& Wellness, WITT, Private Bag 2030, New Plymouth 4342. Email R.Crawford@witt.ac.nz

\section{REFERENCE}

Carpenter, H. M. (197I). An improved system of nursing education for New Zealand. Department of Health, Wellington, New Zealand. 